\title{
ON AXI-SYMMETRICAL VIBRATIONS OF SHALLOW SPHERICAL SHELLS*
}

\author{
BI \\ ERIC REISSNER \\ Massachusetts Institute of Technology
}

1. Introduction. The present note may be considered as a sequel to an earlier paper on the same subject [4]. In this earlier paper the solution of the differential equations for axi-symmetrical vibrations of shallow spherical shells was given in terms of certain Bessel functions. The problem of the frequency determination for a shell segment with clamped edge was considered as an example of application of this solution. It led to the vanishing of a third-order determinant each element of which involved Bessel functions and the solution of a certain cubic (Eqs. 32 and 35 of Ref. 4). Similar results, by a somewhat different method, had earlier been obtained by Federhofer [2]. The Bessel-function frequency determinant being difficult to evaluate, no numerical results have yet been obtained by its use. Instead, an approximate solution for the lowest frequency was obtained by means of the procedure of Rayleigh and Ritz (Equation 40 of Ref. 4 and a similar result in Ref. 2).

The Bessel-function solution of Ref. 4 was obtained on the basis of assumptions which had previously been made for the problem of static deformations of shallow spherical shells [3]. It was not observed at that time that an additional approximation would be appropriate for the problem of transverse vibrations. This additional approximation is based on the fact that for transverse vibrations of shallow shells the magnitude of the longitudinal inertia terms is negligibly small compared with the magnitude of the transverse inertia terms [5]. Upon omission of longitudinal inertia terms it becomes possible to reduce the differential equations of dynamics to the same form as the equations of statics except that the transverse load function must include the d'Alembert term $-\rho h w_{\imath \iota}$.

The present paper contains applications of this result to three specific problems of axi-symmetrical vibrations of spherical shells.

(1) Determination of the lowest frequency of free vibrations for a shell segment with clamped edge. The numerical results are compared with the corresponding results from the earlier Rayleigh-Ritz formula.

(2) Determination of the frequencies of free vibrations of a shell segment with free edge. The relation between the present frequency equation and the corresponding frequency equation for a flat plate is of such nature that known numerical results for the flat plate can be translated with little difficulty so as to furnish the corresponding results for the shallow spherical shell.

(3) Forced vibrations due to point load at apex of the shell. We determine first the pointforce singularity which occurs regardless of the form of the boundary conditions at the edge of the shell. We then consider further the case for which the boundary is sufficiently far removed to assume it at infinity and use the condition that sufficiently far from the point of load application the solution must represent outward travelling waves.

*Received August 10, 1954. A report on work supported by Office of Naval Research under Contract N5-ori-07834 with Massachusetts Institute of Technology. 
Our results generalize corresponding results of $\mathrm{H}$. Cremer and L. Cremer [1] for the unlimited flat plate.

2. Differential equations for transverse vibrations of shallow spherical shells. Let the equation of the shell surface be given by

$$
z=\frac{a^{2}}{2 R}-\frac{x^{2}+y^{2}}{2 R}=\frac{a^{2}-r^{2}}{2 R} .
$$

In the absence of longitudinal loads we have as differential equations for the displacement $w$ in the direction of $z$ and for an Airy stress function $F$,

$$
\begin{gathered}
D \nabla^{2} \nabla^{2} w+\frac{1}{R} \nabla^{2} F=-\rho h \frac{\partial^{2} w}{\partial t^{2}}+p(r, \theta, t) \\
\nabla^{2} \nabla^{2} F-\frac{h E}{R} \nabla^{2} w=0
\end{gathered}
$$

where $\rho=$ density of shell material, $h=$ wall thickness (assumed constant), $E=$ modulus of elasticity, $D=E h^{3} / 12\left(1-\nu^{2}\right)$ and $\nabla^{2}=\partial^{2} / \partial r^{2}+r^{-1} \partial / \partial r+r^{-2} \partial^{2} / \partial \theta^{2}$ (Fig. 1).

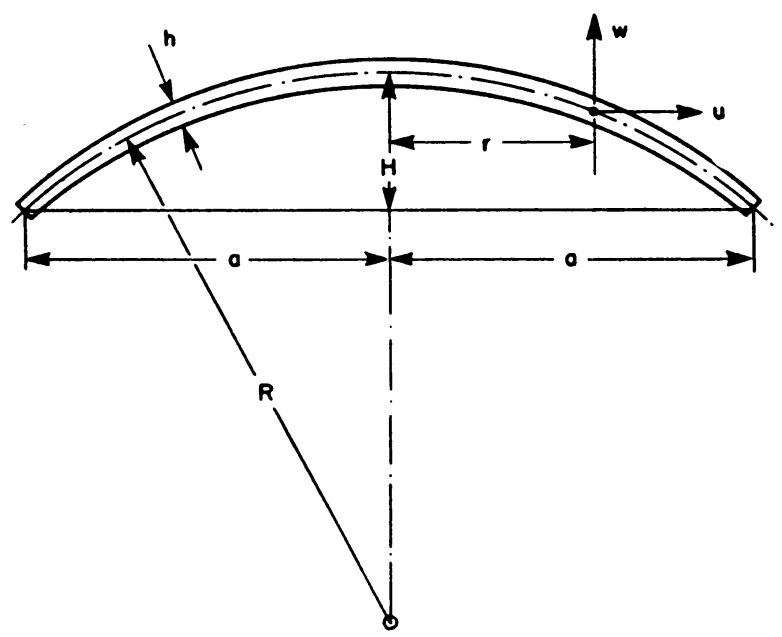

Fig. 1. Spherical shell segment, showing notations for geometrical dimensions.

We shall further need the relations

$$
\begin{gathered}
\epsilon_{\theta}=\frac{N_{\theta}-\nu N_{r}}{E h}, \quad N_{\theta}=\frac{\partial^{2} F}{\partial r^{2}}, \quad N_{r}=\frac{1}{r} \frac{\partial F}{\partial r}+\frac{1}{r^{2}} \frac{\partial^{2} F}{\partial \theta^{2}} \\
M_{r}=-D\left(\frac{\partial^{2} w}{\partial r^{2}}+\frac{\nu}{r} \frac{\partial w}{\partial r}+\frac{\nu}{r^{2}} \frac{\partial^{2} w}{\partial \theta^{2}}\right) \\
Q_{r}=-D \frac{\partial \nabla^{2} w}{\partial r}, \quad V_{r}=Q_{r}+N_{r} \frac{\partial z}{\partial r}+N_{r \theta} \frac{1}{r} \frac{\partial z}{\partial \theta}
\end{gathered}
$$

which are the same as in problems of statics. 
3. Axi-symmetrical solutions. We set

$$
w=e^{i \omega t} W(r), \quad F=e^{i \omega t} f(r), \quad p=e^{i \omega t} q(r) .
$$

Restricting attention to the case $q=0$ it is found that solutions of the homogeneous equations (2) and (3) are of the form

$$
\begin{aligned}
W=C_{1} J_{0}(\lambda r)+C_{2} Y_{0}(\lambda r)+ & C_{3} I_{0}(\lambda r)+C_{4} K_{0}(\lambda r)+C_{5}+C_{6} \log r \\
f=B_{1} J_{0}(\lambda r)+B_{2} Y_{0}(\lambda r)+B_{3} I_{0}(\lambda r)+ & B_{4} K_{0}(\lambda r) \\
& +B_{5} r^{2}+B_{6} r^{2} \log r+C_{7}+C_{8} \log r .
\end{aligned}
$$

The constants $C_{n}$ in (8) and (9) are arbitrary, the usual notation for Bessel functions and modified Bessel functions is employed, and furthermore

$$
\lambda^{4}=\frac{\rho h}{D} \omega^{2}-\frac{h E}{R^{2} D} .
$$

Taking account of the fact that

$$
\nabla^{2}\left(J_{0}, Y_{0}\right)=-\lambda^{2}\left(J_{0}, Y_{0}\right), \quad \nabla^{2}\left(I_{0}, K_{0}\right)=\lambda^{2}\left(I_{0}, K_{0}\right),
$$

there results for the constants $B_{n}$

$$
\left.\begin{array}{rl}
B_{1,2} & =-\frac{h E}{R \lambda^{2}} C_{1,2} \\
B_{3,4} & =\frac{h E}{R \lambda^{2}} C_{3,4} \\
B_{5} & =\frac{1}{4} \rho h \omega^{2} R\left(C_{5}-C_{6}\right) \\
B_{6} & =\frac{1}{4} \rho h \omega^{2} R C_{6}
\end{array}\right\}
$$

Of the eight constants $C_{n}$ only six are physically significant. The constant $C_{z}$ has no effect on stresses and displacements. The condition of vanishing circumferential displacements as in problems of statics [3], is of the form $(h E / R) W=\nabla^{2} f+$ const. From this $(h E / R) C_{6}=4 B_{6}$. With $B_{6}$ from (12) this implies $\lambda^{4} C_{6}=0$. Since $\lambda \neq 0$ we have

$$
B_{6}=C_{6}=0
$$

and therewith altogether

$$
\begin{gathered}
W=C_{1} J_{0}(\lambda r)+C_{2} Y_{0}(\lambda r)+C_{3} I_{0}(\lambda r)+C_{4} K_{0}(\lambda r)+C_{5}, \\
f=-\frac{h E}{R \lambda^{2}}\left[C_{1} J_{0}(\lambda r)+C_{2} Y_{0}(\lambda r)-C_{3} I_{0}(\lambda r)-C_{4} K_{0}(\lambda r)\right]+\frac{1}{4} \rho h \omega^{2} R C_{5} r^{2}+C_{8} \log r .
\end{gathered}
$$

The solution (13) and (14) will be applied to the three problems indicated earlier. 
4. Frequency equation for shell segment with clamped edge. Let $r=a$ be the edge of the shell segment. Since $W$ and $f$ must be regular for $r=0$ we have

$$
C_{2}=C_{4}=C_{8}=0 \text {. }
$$

The conditions of vanishing edge displacement $W$ and edge slope $W^{\prime}$ are

where

$$
\begin{gathered}
C_{1} J_{0}(\mu)+C_{3} I_{0}(\mu)+C_{5}=0, \\
C_{1} J_{0}^{\prime}(\mu)+C_{3} I_{0}^{\prime}(\mu)=0,
\end{gathered}
$$

$$
\mu=\lambda a .
$$

We assume that the third edge condition stipulates vanishing horizontal displacement or, equivalently, vanishing circumferential strain $\epsilon_{\theta}$. With the help of (4) this relation becomes $\left(F^{\prime \prime}-\nu r^{-1} F^{\prime}\right)_{a} \equiv\left[\nabla^{2} F-(1+\nu) r^{-1} F^{\prime}\right]_{a}=0$. Introduction of (14) gives as third relation for the constants $C_{1}, C_{3}, C_{5}$,

$\frac{h E}{R}\left\{C_{1} J_{0}(\mu)+C_{3} I_{0}(\mu)-\frac{1+\nu}{\mu}\left[-C_{1} J_{0}^{\prime}(\mu)+C_{3} I_{0}^{\prime}(\mu)\right]\right\}+\frac{1}{2} \rho h \omega^{2} R(1-\nu) C_{5}=0$.

The frequency equation of the problem is the condition of vanishing of the determinant of the system (16), (17) and (19). Setting $J_{0}^{\prime}=-J_{1}, I_{0}^{\prime}=I_{1}$, this frequency equation may be brought into the following form

$$
J_{0}(\mu) I_{1}(\mu)+J_{1}(\mu) I_{0}(\mu)+\frac{4 \kappa^{4} J_{1}(\mu) I_{1}(\mu)}{\mu\left(\mu^{4}-\kappa^{4}\right)}=0 .
$$

The parameter $\kappa^{4}$ is

$$
\kappa^{4}=\frac{1+\nu}{1-\nu} \frac{h E a^{4}}{R^{2} D}=48(1+\nu)^{2} \frac{H^{2}}{h^{2}}
$$

and $\omega$ is given in terms of $\mu$ and $\kappa$, as follows

$$
\rho \omega^{2} \frac{h a^{4}}{D}=\frac{1-\nu}{1+\nu} \kappa^{4}+\mu^{4} .
$$

The quantity $H$ in (21) is the rise of the shell. It is related to the radii $R$ and $a$ through the formula,

$$
H=\frac{a^{2}}{2 R}
$$

The frequency equation (20) furnishes $\mu$ as a function of $\kappa$. Approximate values of $\mu$ for the lowest frequency may be found in Table $1 .^{*}$ Having $\mu$ as function of $\kappa$ we obtain further from (22)

$$
\omega=\left(\frac{E}{\rho}\right)^{1 / 2} \frac{h}{a^{2}}\left[\frac{\mu^{4}+\kappa^{4}(1-\nu) /(1+\nu)}{12\left(1-\nu^{2}\right)}\right]^{1 / 2} .
$$

Let $\omega_{0}$ be the value of $\omega$ corresponding to $\kappa=0$ and $\nu=0$, that is the value of the frequency $\omega$ for a flat plate and for vanishing Poisson's ratio. We have

$$
\omega_{0}=\left(\frac{E}{\rho}\right)^{1 / 2} \frac{h}{a^{2}}\left(\frac{\mu_{0}^{4}}{12}\right)^{1 / 2}=2.948\left(\frac{E}{\rho}\right)^{1 / 2} \frac{h}{a^{2}},
$$

*For these and all other computations in this paper the author is indebted to Millard W. Johnson. 
TABLE 1. Numerically smallest solution of Eq. (20)

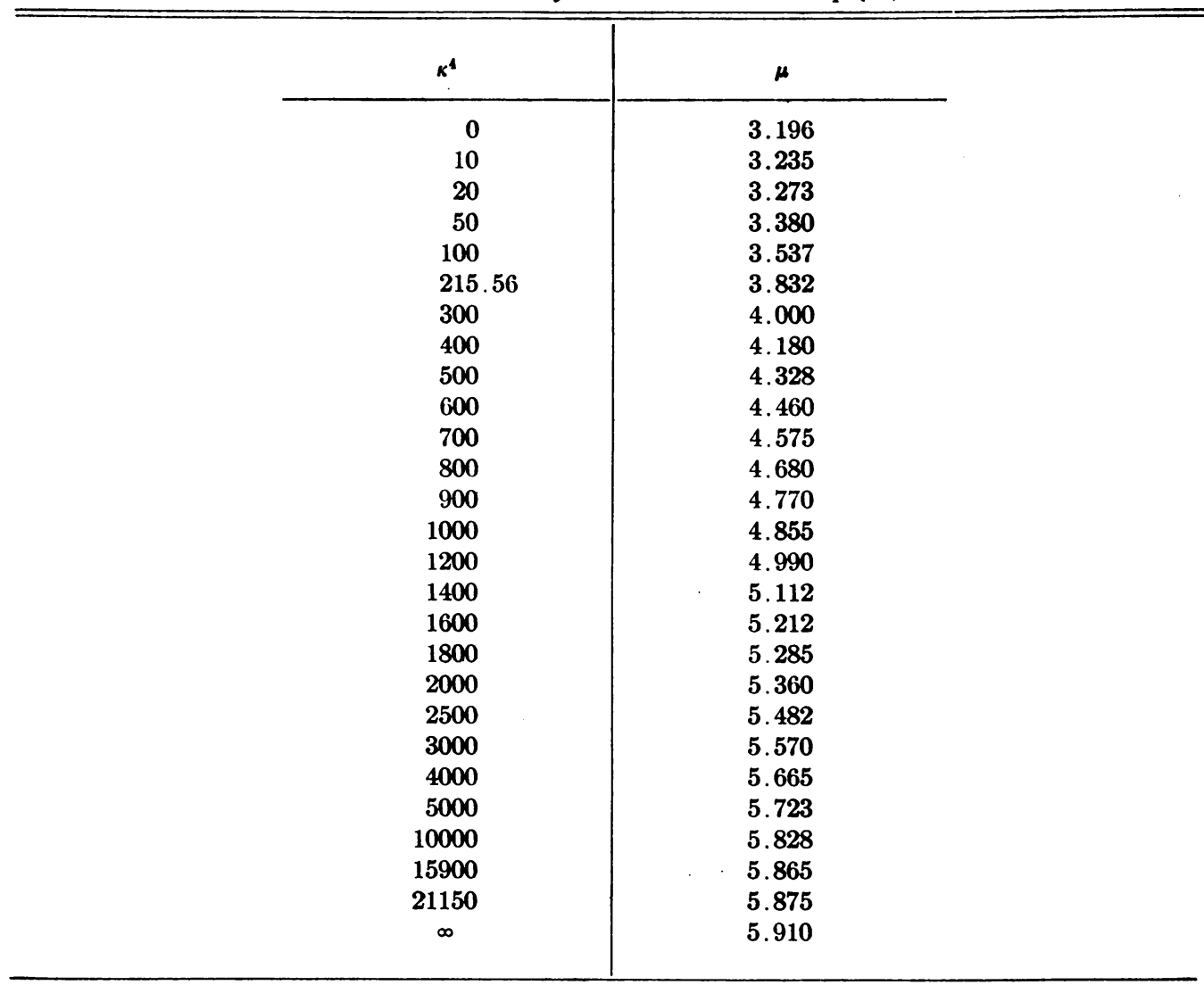

where $\mu_{0}=3.195$ and $\mu_{0}^{4}=104.27$. It is convenient to express the actual values of $\omega$ in units of $\omega_{0}$, as follows,

$$
\frac{\omega}{\omega_{0}}=\left[\frac{1}{1-\nu^{2}}\left(\frac{\mu}{\mu_{0}}\right)^{4}+\frac{48}{\mu_{0}^{4}}\left(\frac{H}{h}\right)^{2}\right]^{1 / 2} .
$$

Numerical values of $\omega / \omega_{0}$ as a function of $\nu$ and $H / h$ may be found in Table II and in Fig. 2.

When $H / h$ is large enough so that the second term under the square root in (26) dominates the first term, practically when $H / h$ is larger than about 25, then Eq. (26) may be replaced by the approximation

$$
\omega=2\left(\frac{E}{\rho}\right)^{1 / 2} \frac{H}{a^{2}} .
$$

It is interesting to note that the thickness $h$ which occurs in the flat-plate frequency formula (25) has been replaced by the shell rise $H$ and the frequency has become independent of wall thickness. It should be emphasized, however, that the simple formula $\left(26^{*}\right)$ depends on two limitations. On the one hand we must have that $H / h \gtrsim 25$ and on the other hand we must have $H / a \lesssim \frac{1}{4}$ in order that the theory of shallow shells is 
TABLE 2. Frequency of clamped-edge shell in units of corresponding flat-plate frequency for $\nu=0$, as function of $\nu$ and $H / h$

\begin{tabular}{|c|c|c|c|}
\hline \multirow{2}{*}{$\frac{H}{h}$} & \multicolumn{3}{|c|}{$\omega / \omega_{0}$} \\
\hline & $\nu=0$ & $\nu=.3$ & $\nu=.5$ \\
\hline 0 & 1.000 & 1.0483 & 1.1547 \\
\hline .5 & 1.08 & 1.149 & 1.28 \\
\hline 1.0 & 1.31 & 1.40 & 1.59 \\
\hline 1.5 & 1.61 & 1.75 & 2.00 \\
\hline 2.0 & 1.94 & 2.16 & 2.42 \\
\hline 2.5 & 2.31 & 2.57 & 2.87 \\
\hline 3.0 & 2.67 & 2.99 & 3. 32 \\
\hline 3.5 & 3.06 & 3.40 & 3.73 \\
\hline 4.0 & 3.43 & 3.78 & 4.12 \\
\hline 4.5 & 3.81 & 4.16 & 4.49 \\
\hline 5 & 4.18 & 4.51 & 4.89 \\
\hline 6 & 4.90 & 5.17 & 5.45 \\
\hline 7 & 5.59 & 5.80 & 6.01 \\
\hline 8 & 6.22 & 6.41 & 6.60 \\
\hline 9 & 6.86 & 7.01 & 7.20 \\
\hline 10 & 7.50 & 7.62 & 7.82 \\
\hline 11 & 8.13 & 8.25 & 8.44 \\
\hline 12 & 8.76 & 8.88 & 9.05 \\
\hline 14 & 10.05 & 10.15 & 10.28 \\
\hline 16 & 11.35 & 11.42 & 11.54 \\
\hline 18 & 12.67 & 12.73 & 12.82 \\
\hline 20 & 14.00 & 14.1 & 14.2 \\
\hline
\end{tabular}

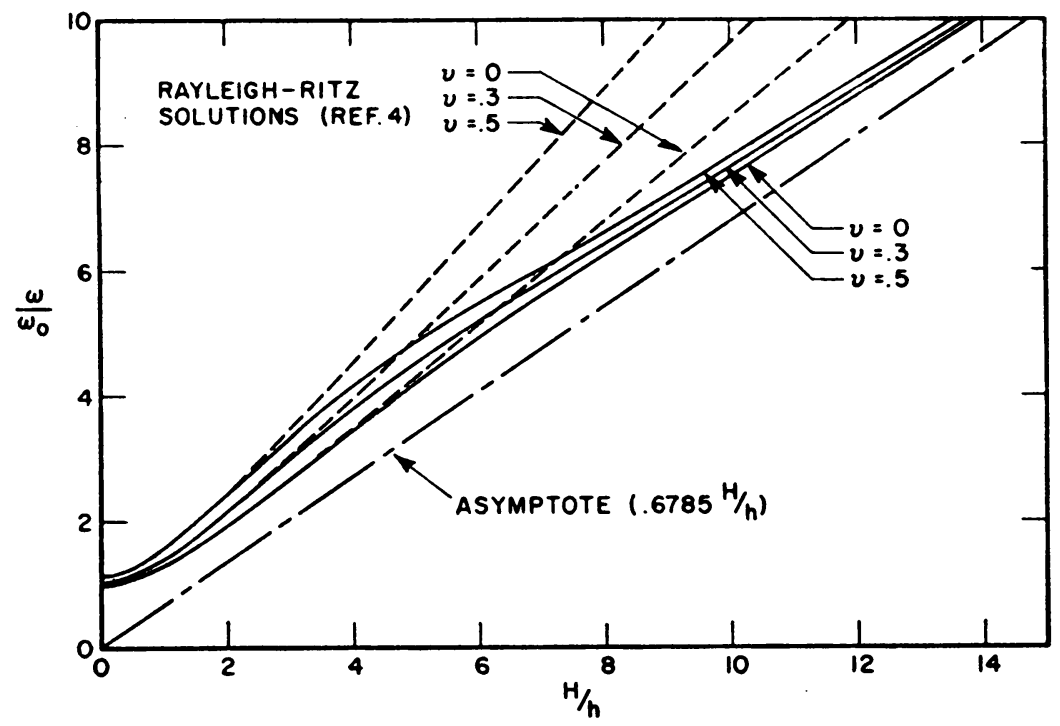

Fig. 2. Lowest frequency for shell segment with clamped edge, in units of corresponding frequency for flat plate with zero Poisson's ratio. 
applicable. We may note that it is this latter restriction which insures that the transverse vibrations of the shell take place at frequencies which are low compared with the frequencies of longitudinal vibrations of flat plates, these latter being of order $(E / \rho)^{1 / 2}(1 / a)$.

Comparison with Rayleigh-Ritz formula. Equation (40) of Ref. 4 may be written in the following form, which is equivalent to (26)

$$
\left[\frac{\omega}{\omega_{0}}\right]_{R R}=\left[\frac{1}{1-\nu^{2}}+\frac{.7+.5 \nu-.2 \nu^{2}}{1-\nu^{2}}\left(\frac{H}{h}\right)^{2}\right]^{1 / 2} .
$$

Figure 2 contains values of $\left(\omega / \omega_{0}\right)_{R R}$ as dotted lines. It is seen that the Rayleigh-Ritz solution agrees remarkably well with the differential equation solution for sufficiently small values of $H / h$, practically up to values of $H / h$ of about three. As $H / h$ increases the error becomes larger, the percentage error approaching a finite limiting value of from 60 per cent to 25 per cent as $\nu$ decreases from 0.5 to 0 .

5. Frequency equation for shell segment with free edge. The boundary conditions for a free edge are

$$
M_{r}=0, \quad V_{r}=0, \quad N_{r}=0,
$$

where $M_{r}, V_{r}$ and $N_{r}$ are given in (4) to (6). To this are again added regularity conditions for $r=0$ which again mean that Eq. (15) must hold. We observe further that (28) contains two relations involving $W^{\prime}, W^{\prime \prime}$ and $W^{\prime \prime \prime}$ but neither $W$ itself nor $f$ and its derivatives. This means that the frequency equation for the shell with free edge does not involve the constant $C_{5}$ in Eq. (13) for $W$. In other words the admissible values of $\mu$ are the same as those for a flat plate with free edge.

The frequency equation for axially symmetric vibrations of a flat plate with free edge, according to Kirchhoff, is

$$
\frac{I_{0}(\mu)}{I_{1}(\mu)}+\frac{J_{0}(\mu)}{J_{1}(\mu)}=\frac{2(1-\nu)}{\mu} .
$$

Examples of the numerically smallest values of $\mu$ are $\nu=0, \mu=2.87 ; \nu=.3, \mu=3.00$; $\nu=.5, \mu=3.07$. Having $\mu$ we obtain $\omega$ by means of (10), (18) and (23) in the form

$$
\omega=\left(\frac{E}{\rho}\right)^{1 / 2}\left[\frac{\mu^{4}}{12\left(1-\nu^{2}\right)} \frac{h^{2}}{a^{4}}+4 \frac{H^{2}}{a^{4}}\right]^{1 / 2} .
$$

Let $\mu_{0}$ and $\omega_{0}$ be appropriate values for the flat plate and for $\nu=0$. We may then write, in analogy to the result (26) for the shell with clamped edge,

$$
\frac{\omega}{\omega_{0}}=\left[\frac{1}{1-\nu^{2}}\left(\frac{\mu}{\mu_{0}}\right)^{4}+\frac{48}{\mu_{0}^{4}}\left(\frac{H}{h}\right)^{2}\right]^{1 / 2} \text {. }
$$

Equation (31) is the same as Eq. (26) except that for the shell with free edge the quantity $\mu$ is independent of the values of $H / h$ while for the shell with clamped edge $\mu$ was found to be a function of $H / h$. Values of $\omega / \omega_{0}$ for the numerically smallest solution of (29) as function of $H / h$ and $\nu$ may be found in Table 3 and Fig. 3 .

We note that when $H / h$ is greater than about 10, Eq. (30) may be simplified to $\omega=2(E / \rho)^{1 / 2}\left(H / a^{2}\right)$ which is the same limiting expression which was previously obtained for the shell segment with clamped edge.

We further note that very probably for this problem of a shell with free edge the 
TABLE 3. Frequency of free-edge shell in units of corresponding flat-plate frequency for $\nu=0$, as function of $\nu$ and $H / h$

\begin{tabular}{|c|c|c|c|}
\hline \multirow{2}{*}{$\frac{H}{h}$} & \multicolumn{3}{|c|}{$\omega / \omega_{0}$} \\
\hline & $\nu=0$ & $\nu=.3$ & $\nu=.5$ \\
\hline 0 & 1 & 1.148 & 1.315 \\
\hline .5 & 1.084 & 1.224 & 1.380 \\
\hline 1.0 & 1.305 & 1.423 & 1.562 \\
\hline 1.5 & 1.610 & 1.705 & 1.821 \\
\hline 2.0 & 1.96 & 2.035 & 2.135 \\
\hline 2.5 & 2.33 & 2.40 & 2.48 \\
\hline 3.0 & 2.71 & 2.77 & 2.85 \\
\hline 3.5 & 3.11 & 3. 16 & 3.22 \\
\hline 4.0 & 3.51 & 3.55 & 3.62 \\
\hline 4.5 & 3.91 & 3.96 & 4.01 \\
\hline 5 & 4.32 & 4.36 & 4.41 \\
\hline 6 & 5.15 & 5.17 & 5.21 \\
\hline 7 & 5.98 & 6.00 & 6.03 \\
\hline 8 & 6.81 & 6.82 & 6.86 \\
\hline 9 & 7.64 & 7.66 & 7.70 \\
\hline 10 & 8.47 & 8.49 & 8.53 \\
\hline 11 & 9.31 & 9.32 & 9.35 \\
\hline 12 & 10.15 & 10.15 & 10.18 \\
\hline 14 & 11.82 & 11.82 & 11.82 \\
\hline 16 & 13.50 & 13.50 & 13.50 \\
\hline 18 & 15.20 & 15.20 & 15.20 \\
\hline 20 & 16.86 & 16.86 & 16.86 \\
\hline
\end{tabular}

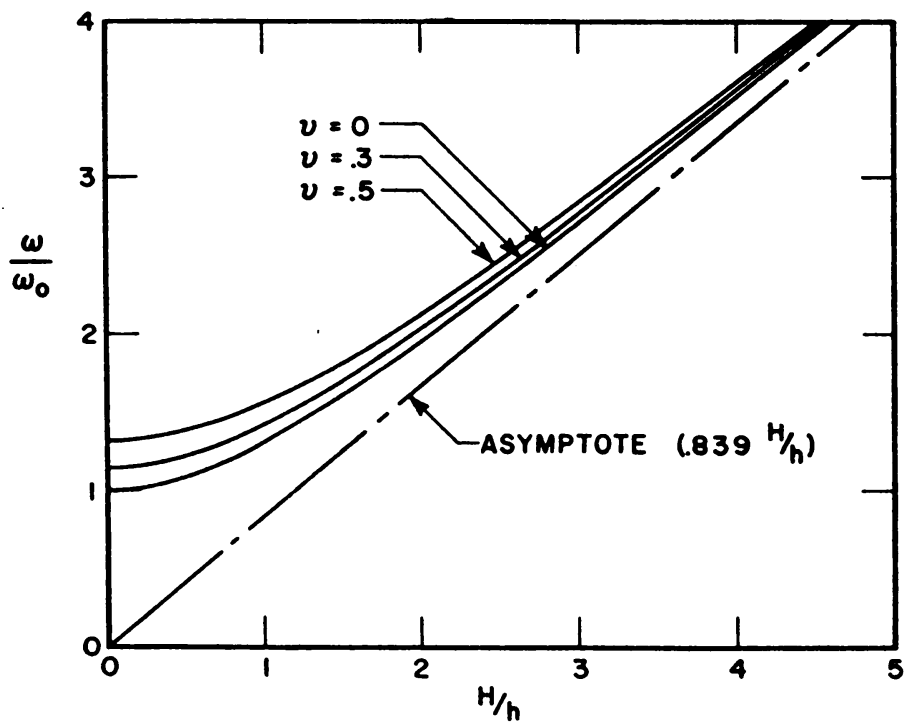

Fig. 3. Lowest frequency of axi-symmetrical vibrations for shell segment with free edge, in units of corresponding frequency for flat plate with zero Poisson's ratio. 
lowest frequency of axi-symmetrical free vibrations is higher than the frequencies of certain non-symmetrical vibrations. This fact is concluded from corresponding known results for the special case of the flat plate.

6. Oscillating point load at apex of shell. The following conditions must be satisfied for an oscillating point load $P \exp (i \omega t)$ at the apex of the shell

$$
\begin{gathered}
\lim _{r \rightarrow 0}\left(2 \pi r V_{r}\right)=P e^{i \omega t}, \\
w(0, t), \quad N_{r}(0, t), \quad N_{\theta}(0, t) \quad \text { finite. }
\end{gathered}
$$

In view of (4), (5), (6), (7) these conditions assume the following form for the solution functions $W$ and $f$ as given by (13) and (14)

$$
\begin{gathered}
\lim _{r \rightarrow 0}\left(r \frac{d \nabla^{2} w}{d r}\right)=-\frac{P}{2 \pi D}, \\
r=0 ; \quad W, \nabla^{2} f, \quad \frac{1}{r} \frac{d f}{d r} \quad \text { finite. }
\end{gathered}
$$

We begin by omitting terms from the solutions (13) and (14) which automatically satisfy the conditions $\left(32^{\prime}\right)$ and $\left(33^{\prime}\right)$ and retain the singular portion

$$
\begin{gathered}
W_{1}=C_{2} Y_{0}(\lambda r)+C_{4} K_{0}(\lambda r), \\
f_{.}=-\frac{h E}{R \lambda^{2}}\left[C_{2} Y_{0}(\lambda r)-C_{4} K_{0}(\lambda r)\right]+C_{8} \log r .
\end{gathered}
$$

Equations (34) and (35) are valid, with real values of the constants $C_{2}$ and $C_{4}$, as long as $\lambda$ is real. According to (10) this means as long as

When

$$
\frac{1}{R}\left(\frac{E}{\rho}\right)^{1 / 2}<\omega .
$$

$$
\omega<\frac{1}{R}\left(\frac{E}{\rho}\right)^{1 / 2}=\frac{2 H}{a^{2}}\left(\frac{E}{\rho}\right)^{1 / 2}
$$

the representations (34) and (35) are no longer convenient. It will, however, be shown that the results obtained in the range of frequencies (36a) are readily transferred to the range (30b).

We note that the frequency which divides the ranges (36a) and (36b) is exactly that lowest frequency of free vibrations which occurs in the range of sufficiently large values of $H / h$ [See Eq. $\left(26^{*}\right)$ ].

In order to determine the constants $C_{2}, C_{4}$ and $C_{8}$ we observe the following relations in which use has been made of (11),

$$
\begin{gathered}
\nabla^{2} W_{1}=\lambda^{2}\left[-C_{2} Y_{0}+C_{4} K_{0}\right], \\
\nabla^{2} f_{\bullet}=\frac{h E}{R}\left[C_{2} Y_{0}+C_{4} K_{0}\right], \\
\frac{1}{r} \frac{d f_{0}}{d r}=-\frac{h E}{R} \frac{1}{\lambda r}\left[C_{2} Y_{0}^{\prime}-C_{4} K_{0}^{\prime}\right]+\frac{C_{8}}{r^{2}}, \\
r \frac{d \nabla^{2} W}{d r}=\lambda^{2}(\lambda r)\left[-C_{2} Y_{0}^{\prime}+C_{4} K_{0}^{\prime}\right],
\end{gathered}
$$




$$
\left.\begin{array}{ll}
Y_{0}(\lambda r) \approx \frac{2}{\pi}\left[\log \frac{\lambda r}{2}+.577 \ldots\right], & Y_{0}^{\prime}(\lambda r) \approx \frac{2}{\pi} \frac{1}{\lambda r} \\
K_{0}(\lambda r) \approx-\left[\log \frac{\lambda r}{2}+.577 \ldots\right], & K_{0}^{\prime}(\lambda r) \approx-\frac{1}{\lambda r}
\end{array}\right\},
$$

Eqs. (38) being valid when $\lambda r \ll 1$.

Introduction of (34), (35), (37) and (38) into the finiteness conditions $\left(33^{\prime}\right)$ shows that these conditions are satisfied provided

$$
\begin{aligned}
& \frac{2}{\pi} C_{2}-C_{4}=0, \\
& \frac{2}{\pi} C_{2}+C_{4}=\frac{\lambda^{2} R}{E h} C_{8} .
\end{aligned}
$$

The load condition $\left(32^{\prime}\right)$ leads to the further relation

$$
\frac{2}{\pi} C_{2}+C_{4}=\frac{P}{2 \pi D \lambda^{2}} .
$$

Introduction of $C_{2}, C_{4}$ and $C_{8}$ from (39) into (34) and (35) leads to the following expressions for the singular solutions $W_{\mathrm{s}}$ and $f_{\mathrm{s}}$,

$$
\begin{gathered}
W_{0}=\frac{P}{4 \pi D \lambda^{2}}\left[\frac{\pi}{2} Y_{0}(\lambda r)+K_{0}(\lambda r)\right] \\
f_{0}=-\frac{E h}{R \lambda^{2}} \frac{P}{4 \pi D \lambda^{2}}\left[\frac{\pi}{2} Y_{0}(\lambda r)-K_{0}(\lambda r)-2 \log r\right] .
\end{gathered}
$$

It will now be shown that (40) and (41) remain valid, but are conveniently written in different form, in the range of small $\omega$ given by (36b). For values of $\omega$ in the range (36b) we write

$$
\lambda=i^{1 / 2}\left[\frac{h E}{R^{2} D}-\frac{\rho h \omega^{2}}{D}\right]^{1 / 4} \equiv i^{1 / 2} \gamma
$$

and we introduce Kelvin functions through the following known relations

$$
\begin{aligned}
& Y_{0}\left(i^{1 / 2} \gamma r\right)=\frac{2}{\pi}[-\operatorname{ker} \gamma r+i \text { kei } \gamma r], \\
& K_{0}\left(i^{1 / 2} \gamma r\right)=\operatorname{ker} \gamma r+i \text { kei } \gamma r .
\end{aligned}
$$

If (42) and (43) are introduced into (40) and (41) there follow as expressions for $W$. and $f$, which are valid when $\omega<R^{-1}(E / \rho)^{1 / 2}$,

$$
\begin{gathered}
W_{\bullet}=\frac{P}{2 \pi D \gamma^{2}} \text { kei } \gamma r, \\
f_{\text {。 }}=-\frac{E h}{R \gamma^{2}} \frac{P}{2 \pi D \gamma^{2}}[\operatorname{ker} \gamma r+\log r] .
\end{gathered}
$$

Equations (44) and (45) contain as special case, when $\omega=0$, the previously given formulas [3, Eq. (48)] for the corresponding problem of static deflection. Writing 


$$
\begin{gathered}
\gamma=\left(h E / R^{2} D\right)^{1 / 4}=\left[12\left(1-\nu^{2}\right)\right]^{-1 / 4}(R h)^{-1 / 2} \text { Eqs. (44) and (45) become for } \omega=0 \\
W_{\star}=\frac{\left[12\left(1-\nu^{2}\right)\right]^{1 / 2}}{2 \pi} \frac{P R}{E h} \mathrm{kei} \frac{r}{\left[12\left(1-\nu^{2}\right)\right]^{1 / 4}(R h)^{1 / 2}}, \\
f_{.}=-\frac{P R}{2 \pi}\left[\operatorname{ker} \frac{r}{\left[12\left(1-\nu^{2}\right)\right]^{1 / 4}(R h)^{1 / 2}}+\log r\right] .
\end{gathered}
$$

We finally note the following important fact. As long as the solution (44) applies, that is as long as $\omega<\left(2 H / a^{2}\right)(E / \rho)^{1 / 2}$, we have that the deflection amplitude $W$ decreases exponentially at large distances $r$ from the point of load application. When $\omega$ is larger than $\left(2 H / a^{2}\right)(E / \rho)^{1 / 2}$ so that the solution (40) applies then the deflection amplitude $W$ decreases as $r^{-1 / 2}$, that is, much more slowly than exponentially. In this latter case an important distinction can be made between standing wave and travelling wave solutions.

7. Travelling wave solution for effectively unlimited shell segment. We inquire for a solution in which the deflection $w=\exp (i \omega t) W(r)$ behaves for sufficiently large values of $r$ as $r^{-1 / 2} \exp [i(\omega t-\lambda r)]$. For a solution with this type of behavior we have that the wave produced by the pulsating point load travels outward, with energy being dissipated through radiation.

In view of the asymptotic behavior of the Hankel function of the second kind

$$
H_{0}^{(2)}(\lambda r)=J_{0}(\lambda r)-i Y_{0}(\lambda r) \sim\left(\frac{2}{\pi \lambda r}\right)^{1 / 2} \exp [-i(\lambda r-\pi / 4)],
$$

an appropriate result is obtained by adding in (40) and (41) a suitable multiple of the non-singular solution $J_{0}(\lambda r)$ as follows,

$$
\begin{gathered}
W_{S T}=\frac{P}{4 \pi D \lambda^{2}}\left\{\frac{\pi}{2}\left[Y_{0}(\lambda r)+i J_{0}(\lambda r)\right]+K_{0}(\lambda r)\right\}, \\
f_{S T}=-\frac{E h}{R \lambda^{2}} \frac{P}{4 \pi D \lambda^{2}}\left\{\frac{\pi}{2}\left[Y_{0}(\lambda r)+i J_{0}(\lambda r)\right]-K_{0}(\lambda r)-2 \log r\right\} .
\end{gathered}
$$

We note that the solution (47) and (48) contains as a special case the corresponding result for a flat plate, as given by $\mathrm{H}$. Cremer and L. Cremer [1]. The case of the flat plate follows if we set $R=\infty$ in the expression for $\lambda$, making $\lambda=\left(\rho h \omega^{2} / D\right)^{1 / 4}$. We note further that while for the spherical shell the solution (47) and (48) is valid, subject to the restriction that $\omega>(E / \rho)^{1 / 2} R$, this relation ceases to be a restriction for the case of the flat plate.

In order that the travelling wave solution (47) have a meaning it is necessary that the boundary $r=a$ be sufficiently far removed from the point of load application. Let us assume that sufficiently far means

$$
\alpha \lambda>5, \quad \text { say. }
$$

Equation (49) may be written as a restriction on frequencies as follows

$$
\omega>\frac{2 H}{a^{2}}\left(\frac{E}{\rho}\right)^{1 / 2}\left[1+\frac{625}{48\left(1-\nu^{2}\right)} \frac{h^{2}}{H^{2}}\right]^{1 / 2} \text {. }
$$

8. Two formulas of acoustical significance. We consider the ratio of velocity at the point of load application to the force causing this velocity, $(\partial w / \partial t)_{r-0} / P \exp (i \omega t)$. 
According to Eq. (47)

$$
\frac{(\partial w / \partial t)_{r=0}}{P e^{i \omega t}}=\frac{i \omega}{4 \pi D \lambda^{2}} \lim _{r \rightarrow 0}\left\{\frac{\pi}{2}\left[Y_{0}(\lambda r)+i J_{0}(\lambda r)\right]+K_{0}(\lambda r)\right\} .
$$

Since $J_{0}(0)=1$ and, according to (38), $\lim _{r \rightarrow 0}\left[\frac{1}{2} \pi Y_{0}(\lambda r)+K_{0}(\lambda r)\right]=0$ there follows,

$$
\frac{(\partial \omega / \partial t)_{r=0}}{P e^{i \omega t}}=\frac{-\omega}{8 D \lambda^{2}}=\frac{-\omega}{8\left[\rho h D\left(\omega^{2}-4 \frac{E}{\rho} \frac{H^{2}}{a^{4}}\right)\right]^{1 / 2}}, \quad \frac{2 H}{a^{2}}\left(\frac{E}{\rho}\right)^{1 / 2}<\omega .
$$

For $H=0$, Eq. (50a) reduces to a result in Ref. 1. We note that a ratio which is independent of frequency for the flat plate is a function of frequency for the shallow spherical shell. In the range of applicability of (50a), which is given by $\left(49^{\prime}\right)$, this ratio decreases with increasing frequency towards a limiting value which is the constant flat plate value.

When $\omega<\left(2 H / a^{2}\right)(E / \rho)^{1 / 2}$ so that Eq. (44) applies we have instead of (50a)

$$
\frac{(\partial w / \partial t)_{r-0}}{P e^{i \omega t}}=\frac{i \omega \mathrm{kei}(0)}{2 \pi D \gamma^{2}} \text {. }
$$

In view of the fact that $\operatorname{kei}(0)=-\frac{1}{4} \pi$ and with $\gamma$ defined in (42) this may be written in the following form

$$
\frac{(\partial w / \partial t)_{r=0}}{P e^{i \omega t}}=\frac{-i \omega}{8\left[\rho h D\left(4 \frac{E}{\rho} \frac{H^{2}}{a^{4}}-\omega^{2}\right)\right]^{1 / 2}}, \quad \frac{2 H}{a^{2}}\left(\frac{E}{\rho}\right)^{1 / 2} .
$$

We finally observe, on the basis of (50a) and (50b) that the following result holds for the work of the force $P \exp (i \omega t)$ per cycle

$$
\frac{\text { Work }}{\text { Cycle }}=\left\{\begin{array}{cl}
0, & \omega<\frac{2 H}{a^{2}}\left(\frac{E}{\rho}\right)^{1 / 2}, \\
\frac{P^{2}}{4\left[\rho h D\left(\omega^{2}-4 \frac{E}{\rho} \frac{H^{2}}{a^{4}}\right)\right]^{1 / 2}}, & \frac{2 H}{a^{2}}\left(\frac{E}{\rho}\right)^{1 / 2}<\omega .
\end{array}\right.
$$

It is recalled that Eq. (51) is derived without consideration of damping sources other than radiation damping. In addition to this, while formally the second part of Eq. (51) holds for all $\omega$ greater than $\left(2 H / a^{2}\right)(E / \rho)^{1 / 2}$, for the solution to have physical meaning a stronger restriction such as $\left(49^{\prime}\right)$ actually applies.

\section{REFERENCES}

1. H. and L. Cremer, Theorie der Entstehung des Klopfschalls, Z. f. Schwingungs-und Schwachstromtechnik 2, 61-72 (1948)

2. K. Federhofer, Zur Berechnung der Eigenschwingungen der Kugelschale, Sitzber. Akad. Wiss. Wien $146,57-69$ (1937)

3. E. Reissner, Stresses and small displacements of shallow spherical shells, J. Math. and Phys. 25, 80-85, 279-300 (1945); 27, 240 (1948)

4. E. Reissner, On vibrations of shallow spherical shells, J. Appl. Phys. 17, 1038-1042 (1946)

5. E. Reissner, On transverse vibrations of thin shallow elastic shells, Q. Appl. Math. 13, 169-176 (1955). 